A fully implicit Newton-Krylov-Schwarz method for tokamak magnetohydrodynamics: Jacobian construction and preconditioner formulation

This article has been downloaded from IOPscience. Please scroll down to see the full text article.

2012 Comput. Sci. Disc. 5014003

(http://iopscience.iop.org/1749-4699/5/1/014003)

View the table of contents for this issue, or go to the journal homepage for more

Download details:

IP Address: 129.119.144.48

The article was downloaded on 08/06/2012 at 15:38

Please note that terms and conditions apply. 


\title{
CONPUTATIONAL
}

\section{A fully implicit Newton-Krylov-Schwarz method for tokamak magnetohydrodynamics: Jacobian construction and preconditioner formulation}

\author{
Daniel R Reynolds ${ }^{1}$, Ravi Samtaney ${ }^{2}$ and Hilari C Tiedeman ${ }^{1}$ \\ ${ }^{1}$ Department of Mathematics, Southern Methodist University, Dallas, TX 75275-0156, USA \\ ${ }^{2}$ Mechanical Engineering, Division of Physical Science and Engineering, King Abdullah \\ University of Science and Technology, Thuwal, Saudi Arabia \\ E-mail: reynolds@smu.edu, Ravi.Samtaney@kaust.edu.sa and htiedeman@smu.edu
}

Received 9 November 2011, in final form 20 April 2012

Published 7 June 2012

Computational Science \& Discovery 5 (2012) 014003 (17pp)

doi:10.1088/1749-4699/5/1/014003

\begin{abstract}
Single-fluid resistive magnetohydrodynamics (MHD) is a fluid description of fusion plasmas which is often used to investigate macroscopic instabilities in tokamaks. In MHD modeling of tokamaks, it is often desirable to compute MHD phenomena to resistive time scales or a combination of resistive-Alfvén time scales, which can render explicit time stepping schemes computationally expensive. We present recent advancements in the development of preconditioners for fully nonlinearly implicit simulations of singlefluid resistive tokamak MHD. Our work focuses on simulations using a structured mesh mapped into a toroidal geometry with a shaped poloidal cross-section, and a finite-volume spatial discretization of the partial differential equation model. We discretize the temporal dimension using a fully implicit $\theta$ or the backwards differentiation formula method, and solve the resulting nonlinear algebraic system using a standard inexact Newton-Krylov approach, provided by the sunDIALs library. The focus of this paper is on the construction and performance of various preconditioning approaches for accelerating the convergence of the iterative solver algorithms. Effective preconditioners require information about the Jacobian entries; however, analytical formulae for these Jacobian entries may be prohibitive to derive/implement without error. We therefore compute these entries using automatic differentiation with OPENAD. We then investigate a variety of preconditioning formulations inspired by standard solution approaches in modern MHD codes, in order to investigate their utility in a preconditioning context. We first describe the code modifications necessary for the use of the OPENAD tool and sUNDIALS solver library. We conclude with numerical results for each of our preconditioning approaches in the context of pellet-injection fueling of tokamak plasmas. Of these, our optimal approach results in a speedup of a factor of 3 compared with non-preconditioned implicit tests, with that performance gap rapidly widening with increasing mesh refinement.
\end{abstract}




\section{Contents}

1. Introduction 2

2. Model

3. Solver framework $\quad 4$

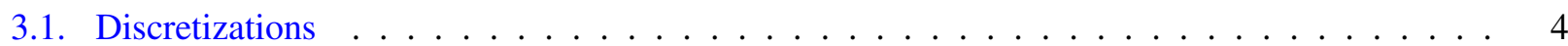

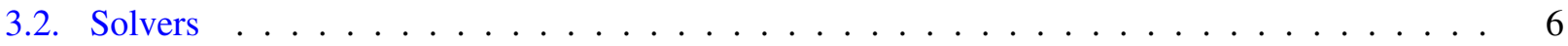

4. Jacobian construction $\quad 6$

5. Preconditioner formulations $\quad 8$

5.1. Alternating direction implicit split preconditioner, $P_{\mathrm{ADI}} \ldots \ldots \ldots \ldots$

5.2. Restricted additive Schwarz preconditioners . . . . . . . . . . . . . . . . . . . 9

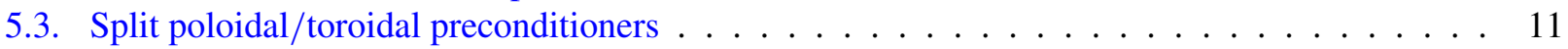

6. Numerical results $\quad 11$

$\begin{array}{ll}\text { 7. Conclusions and future work } & 15\end{array}$

$\begin{array}{lr}\text { Acknowledgments } & 16\end{array}$

$\begin{array}{ll}\text { References } & 16\end{array}$

\section{Introduction}

Single-fluid resistive magnetohydrodynamics (MHD) is a fluid description of a plasma that is frequently employed to investigate macroscopic instabilities in tokamaks. In MHD modeling of tokamaks, it is often desirable to compute MHD phenomena to resistive time scales or a combination of resistive-Alfvén time scales, which makes explicit time stepping schemes prohibitively expensive. We present recent advancements in the development of preconditioners for fully nonlinearly implicit simulations of singlefluid visco-resistive tokamak MHD. Our work focuses on simulations using a structured mesh mapped into a toroidal geometry with a shaped poloidal cross-section and a finite-volume discretization of the spatial derivatives in the visco-resistive MHD model. We discretize in time using either a fully implicit $\theta$ or the backwards differentiation formula (BDF) method. We solve the resulting nonlinear algebraic system using an inexact Newton-Krylov approach, through the sunDiaLs library of nonlinear solvers. In this solver, all Jacobian-vector products are computed in 'matrix-free' mode, using the underlying physical flux routines to generate finite-difference approximations to the directional derivatives used within the Krylov method. This basic implicit solver infrastructure has been described previously in $[1,2]$.

True algorithmic scalability of inexact Newton methods with increasing problem size requires an effective preconditioner for accelerating the convergence of the inner Krylov solver. Typically, effective preconditioners require information about the Jacobian entries, to be used within some approximate solution strategy. Analytical formulae for these Jacobian entries may be difficult to derive and implement without error, especially so if nonlinear upwind methods or complex boundary conditions are used. To this end, we have employed the OPENAD tool to compute these derivatives automatically. With these Jacobian entries, we investigate a variety of preconditioning approaches, based on standard solution algorithms in modern MHD codes, in order to better understand their efficacy as preconditioners for fully implicit formulations.

We note that the topic of implicit solvers for tokamak MHD has been studied for some time. A recent review article by Jardin [3] provides an excellent historical account of the evolution of such approaches, starting with semi-implicit formulations that implicitly treated only the stiffest components, to the more recent development of full nonlinearly implicit approaches. In the context of fully implicit approaches for three-dimensional (3D) MHD, we point out the recent success of physics-based preconditioning techniques by Chacón in a Cartesian slab geometry [4]. Additionally, there have been a number of studies using fully implicit simulations in tokamak geometries, including an approach by Breslau and Jardin that utilized alternating direction implicit (ADI) solvers for the linear Newton systems [5], as well as a very interesting approach by Lütjens and Luciani that employed an operator-split preconditioner within a matrix-free Newton 
Krylov solver [6]. However, it is our belief that the present work is the first approach employing fully implicit simulations in a tokamak geometry to also provide a thorough investigation of the effect of various preconditioner approximations on the parallel scaling performance of the overall implicit algorithm.

We first describe our partial differential equation (PDE) model for visco-resistive MHD in section 2. We then summarize our implicit solver framework in section 3. The core of this paper focuses on our new approaches for the construction of Jacobian entries and the formulation of geometry-based preconditioners, in sections 4 and 5 . We then present the results of tests of these preconditioning approaches in section 6.

\section{Model}

We consider the visco-resistive MHD equations in cylindrical, $(r, \varphi, z)$, coordinates,

$$
\partial_{t} \mathbf{U}+\frac{1}{r} \partial_{r}(r \mathbf{F}(\mathbf{U}))+\partial_{z} \mathbf{H}(\mathbf{U})+\frac{1}{r} \partial_{\varphi} \mathbf{G}(\mathbf{U})=\mathbf{S}(\mathbf{U})+\nabla \cdot \mathbf{F}_{\mathrm{d}}(\mathbf{U}),
$$

where $\mathbf{U}=\left(\rho, \rho u_{r}, \rho u_{\varphi}, \rho u_{z}, B_{r}, B_{\varphi}, B_{z}, e\right)$, with $\rho$ the density, $\mathbf{u}=\left(u_{r}, u_{\varphi}, u_{z}\right)$ the velocity, $\mathbf{B}$ the magnetic induction and $e$ the total energy. The hyperbolic fluxes are given by

$$
\begin{aligned}
& \mathbf{F}=\begin{array}{c}
\left(\rho u_{r}, \rho u_{r}^{2}+\tilde{p}-B_{r}^{2}, \rho u_{r} u_{\varphi}-B_{r} B_{\varphi}, \rho u_{r} u_{z}-B_{r} B_{z}, 0,\right. \\
\left.u_{r} B_{\varphi}-u_{\varphi} B_{r}, u_{r} B_{z}-u_{z} B_{r},(e+\tilde{p}) u_{r}-(\mathbf{B} \cdot \mathbf{u}) B_{r}\right),
\end{array} \\
& \mathbf{G}=\begin{array}{c}
\left(\rho u_{\varphi}, \rho u_{r} u_{\varphi}-B_{r} B_{\varphi}, \rho u_{\varphi}^{2}+\tilde{p}-B_{\varphi}^{2}, \rho u_{z} u_{\varphi}-B_{z} B_{\varphi}\right. \\
\left.u_{\varphi} B_{r}-u_{r} B_{\varphi}, 0, u_{\varphi} B_{z}-u_{z} B_{\varphi},(e+\tilde{p}) u_{\varphi}-(\mathbf{B} \cdot \mathbf{u}) B_{\varphi}\right)
\end{array} \\
& \mathbf{H}=\begin{array}{c}
\left(\rho u_{z}, \rho u_{r} u_{z}-B_{r} B_{z}, \rho u_{z} u_{\varphi}-B_{z} B_{\varphi}, \rho u_{z}^{2}+\tilde{p}-B_{z}^{2},\right. \\
\left.u_{z} B_{r}-u_{r} B_{z}, u_{z} B_{\varphi}-u_{\varphi} B_{z}, 0,(e+\tilde{p}) u_{z}-(\mathbf{B} \cdot \mathbf{u}) B_{z}\right),
\end{array}
\end{aligned}
$$

where $\tilde{p}=p+\frac{\mathbf{B} \cdot \mathbf{B}}{2}$ and $e=\frac{p}{\gamma-1}+\frac{\rho \mathbf{u} \cdot \mathbf{u}}{2}+\frac{\mathbf{B} \cdot \mathbf{B}}{2} . \mathbf{S}(\mathbf{U})$ is a local source term resulting from the cylindrical coordinate system,

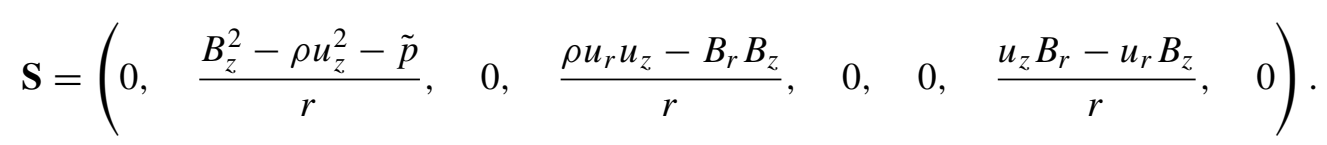

Note that $\nabla \cdot \mathbf{F}_{\mathrm{d}}(\mathbf{U})$ correspondingly incorporates diffusive components of the visco-resistive model,

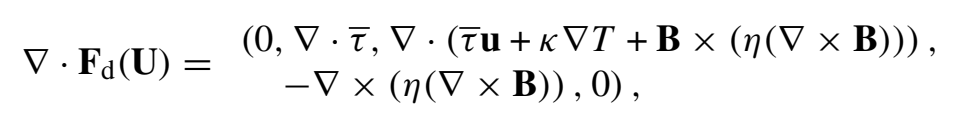

with viscous stress tensor $\bar{\tau}=\mu\left(\nabla \mathbf{u}+(\nabla \mathbf{u})^{T}\right)-\frac{2}{3} \mu(\nabla \cdot \mathbf{u}) \bar{I}$, and temperature $T=\frac{p}{\rho R_{\text {gas }}}$. Input parameters include the viscosity $\mu$, the resistivity $\eta$ and the heat conduction coefficient $\kappa$.

To capture the toroidal tokamak geometry, we map the cylindrical $(r, \varphi, z)$ computational domain into a $(\xi, \varphi, \eta)$ physical domain, as shown in figure 1 . We note that this mapping only involves the coordinates $(r, z)$ and $(\xi, \eta)$ within the poloidal plane,

$$
\begin{array}{llll}
\xi=\xi(r, z), & \eta=\eta(r, z), & \varphi=\varphi & \text { (cylindrical } \rightarrow \text { mapped) } \\
r=r(\xi, \eta), & z=z(\xi, \eta), & \varphi=\varphi & \text { (mapped } \rightarrow \text { cylindrical). }
\end{array}
$$

These mappings have Jacobian determinants

$$
\mathcal{J}=\left(\partial_{\xi} r\right)\left(\partial_{\eta} z\right)-\left(\partial_{\eta} r\right)\left(\partial_{\xi} z\right) \quad \text { and } \quad \mathcal{J}^{-1}=\left(\partial_{r} \xi\right)\left(\partial_{z} \eta\right)-\left(\partial_{r} \eta\right)\left(\partial_{z} \xi\right)
$$




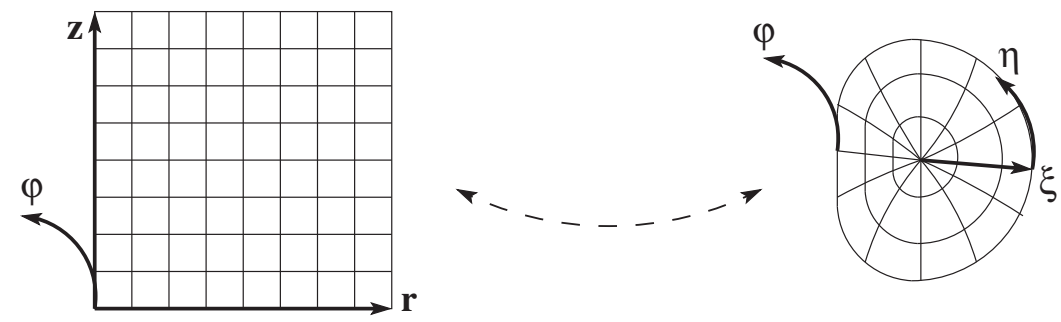

Figure 1. Mapping between the cylindrical computational grid and the toroidal physical domain.
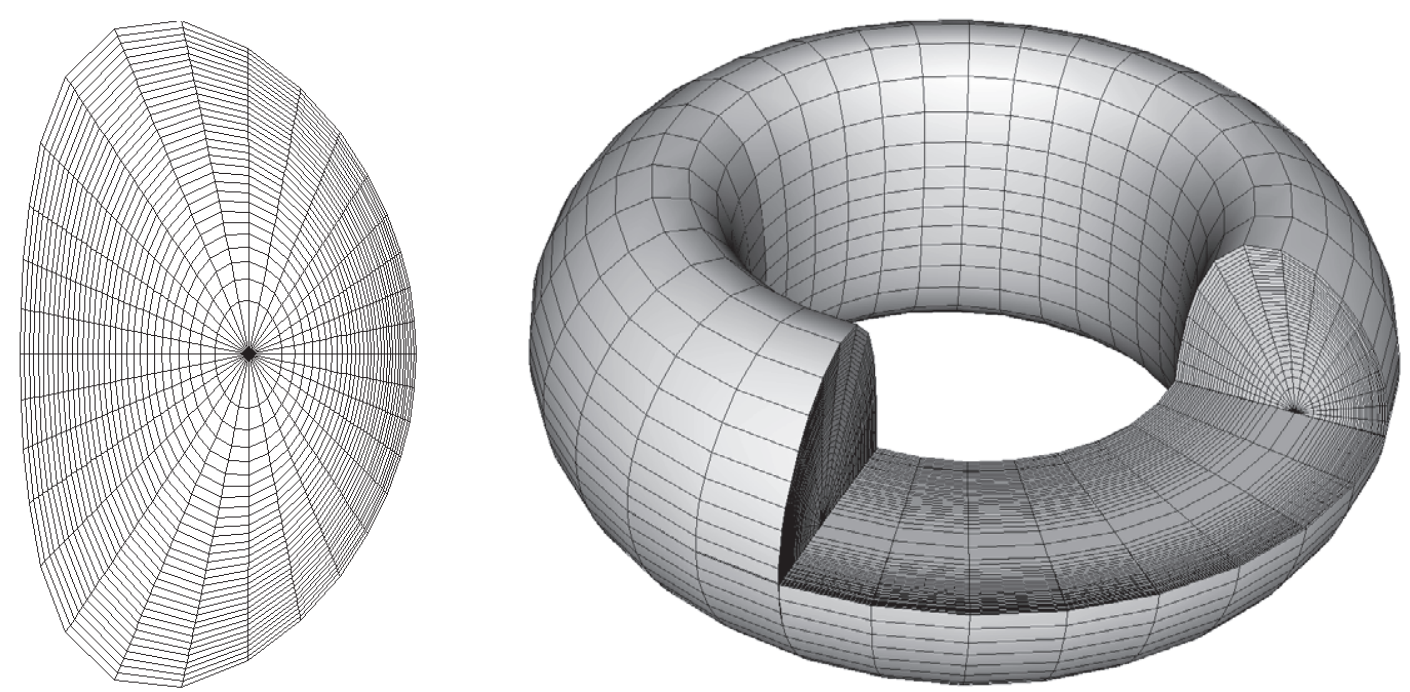

Figure 2. Spatial meshes. Left: $2 \mathrm{D}$ mesh covering the $(\xi, \eta)$ poloidal plane. Right: 3D mesh, with slice removed to show grid structure.

With this mapping, we rewrite (1) in the tokamak domain as

$$
\partial_{t} \mathbf{U}+\frac{1}{r \mathcal{J}}\left[\partial_{\xi}(r \tilde{\mathbf{F}}(\mathbf{U}))+\partial_{\eta}(r \tilde{\mathbf{H}}(\mathbf{U}))+\partial_{\varphi}(\tilde{\mathbf{G}}(\mathbf{U}))\right]=\mathbf{S}(\mathbf{U})+\nabla \cdot \tilde{\mathbf{F}}_{\mathrm{d}}(\mathbf{U})
$$

with the modified fluxes

$$
\begin{aligned}
& \tilde{\mathbf{F}}=\mathcal{J}\left[\left(\partial_{r} \xi\right) \mathbf{F}+\left(\partial_{z} \xi\right) \mathbf{H}\right]=\left(\partial_{\eta} z\right) \mathbf{F}-\left(\partial_{\eta} r\right) \mathbf{H}, \\
& \tilde{\mathbf{H}}=\mathcal{J}\left[\left(\partial_{r} \eta\right) \mathbf{F}+\left(\partial_{z} \eta\right) \mathbf{H}\right]=\left(\partial_{\xi} z\right) \mathbf{F}-\left(\partial_{\xi} r\right) \mathbf{H} \text { and } \\
& \tilde{\mathbf{G}}=\mathcal{J} \mathbf{G}
\end{aligned}
$$

with similar transformations required for the diffusive terms, $\nabla \cdot \tilde{\mathbf{F}}_{\mathrm{d}}(\mathbf{U})$.

We further note that 2D simulations in the $(\xi, \eta)$ plane are straightforward with this model (10), and only assume uniformity in the toroidal direction, i.e. $\partial_{\varphi} \mathbf{U}=0$.

\section{Solver framework}

\subsection{Discretizations}

We approximate (10) in space using a second-order finite-volume discretization, in which the domain is decomposed into a structured grid of cells, with all unknowns $\mathbf{U}$ located at the cell centers. Typical discretizations in two and three dimensions are shown in figure 2. Due to the coordinate mixing between $(r, z)$ 

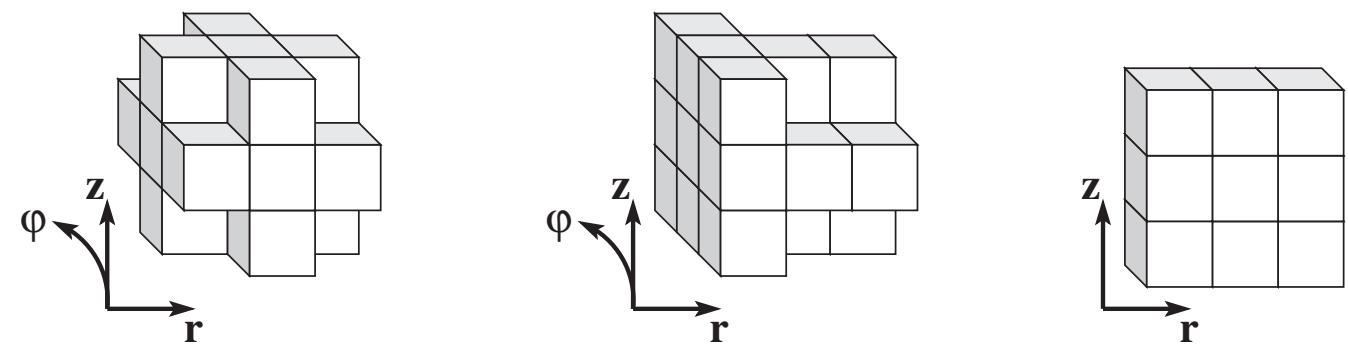

Figure 3. Stencils used in calculations: full 19-point 3D stencil within domain interior (left), modified 19-point 3D stencil at $r$-left domain boundary (middle) and full 9-point 2D stencil (right).

and $(\xi, \eta)$ in the mapped fluxes (11) and (12), this second-order discretization results in a 19-point nearestneighbor stencil. Moreover, at the domain boundaries $\xi=\xi_{\min }$ and $\xi=\xi_{\max }$, second-order accuracy requires that the centered stencil be modified to a one-sided 19-point approximation. For the 2D model, second-order differencing requires a 9-point stencil. Examples of these stencils are shown in figure 3.

Under a method-of-lines approach, we condense notation to write this spatially semi-discretized equation (10) as

$$
\partial_{t} \mathbf{U}=\mathcal{R}(\mathbf{U}),
$$

where we now use $\mathbf{U}$ to denote the vector of discrete solution values over the domain. The hyperbolic fluxes $\tilde{\mathbf{F}}, \tilde{\mathbf{H}}$ and $\tilde{\mathbf{G}}$ exhibit a wide disparity of wave speeds, spanning up to two to three orders of magnitude. In addition, the second spatial derivatives within $\nabla \cdot \mathbf{F}_{\mathrm{d}}$ lead to increasingly prohibitive explicit stability restrictions. Moreover, the compressible MHD system permits the fast compressive wave, which can have speeds two orders of magnitude faster than the typical flow speed of the plasma. Hence the visco-resistive MHD equations are emblematic of a stiff PDE system, in which the dynamics of interest evolve on a much slower time scale than that prescribed for numerical stability considerations alone. We therefore employ a fully implicit discretization in time to ensure numerical stability when evolving at time steps of interest that may exceed stability-restricted time steps for explicit methods. To this end, we use one of two time discretization approaches. The simpler approach employs a $\theta$-method to move between time steps $t^{n}$ and $t^{n+1}$,

$$
\mathbf{U}^{n+1}-\mathbf{U}^{n}-\Delta t^{n+1}\left[\theta \mathcal{R}\left(\mathbf{U}^{n+1}\right)+(1-\theta) \mathcal{R}\left(\mathbf{U}^{n}\right)\right]=0,
$$

where $\Delta t^{n+1}=t^{n+1}-t^{n}$, that allows for either first-order $(0.5<\theta \leqslant 1)$ or second-order $(\theta=0.5)$ accurate fully implicit simulations. For $0.5 \leqslant \theta \leqslant 1$ the method is A-stable, and for $\theta=1$ it is L-stable.

Our more advanced time integration strategy employs the variable-step and variable-order cvoDE solver from the sundials library [7]. This approach utilizes a fully implicit linear multistep method based on the fixed-leading-coefficient BDF formulae,

$$
\mathbf{U}^{n+1}-\beta_{0} \Delta t^{n+1} \mathcal{R}\left(\mathbf{U}^{n+1}\right)-\sum_{i=1}^{q}\left[\alpha_{i} \mathbf{U}^{n-i}+\beta_{i} \Delta t^{n+1} \mathcal{R}\left(\mathbf{U}^{n-i}\right)\right]=0,
$$

where $1 \leqslant q \leqslant 5$ corresponds to the order of accuracy. For $q \in\{1,2\}$ this method is A- and L-stable, and for higher orders it is $A(\alpha)$ stable with angle $\alpha$ shrinking as $q$ increases.

In both cases, if we define $\mathbf{g}$ as a vector of the data available from previous time solutions, and $\gamma$ as either $\theta \Delta t^{n+1}$ or $\beta_{0} \Delta t^{n+1}$, the implicit time-discretized system may be written in the form

$$
\mathbf{f}(\mathbf{U}) \equiv \mathbf{U}-\gamma \mathcal{R}(\mathbf{U})-\mathbf{g}=0 .
$$

As a result, each time step in the evolution of our system (10) is performed by solving the nonlinear algebraic system (17). 


\subsection{Solvers}

We solve equation (17) using inexact Newton-Krylov methods from the sunDIALs library, as described in $[1,2,7]$. These are based on the Newton iteration for solving $\|\mathbf{f}(\mathbf{U})\|<\epsilon, \mathbf{U}_{k+1}=\mathbf{U}_{k}+\mathbf{s}_{k}$, where the Newton update $\mathbf{s}_{k}$ is found through solving the linear system,

$$
J\left(\mathbf{U}_{k}\right) \mathbf{s}_{k}=-\mathbf{f}\left(\mathbf{U}_{k}\right), \quad \text { where } J\left(\mathbf{U}_{k}\right) \equiv \frac{\partial \mathbf{f}}{\partial \mathbf{U}}\left(\mathbf{U}_{k}\right) .
$$

Inexact Newton-Krylov methods do not solve this linear system exactly, and instead approximate the solutions to (18) by solving the problem

$$
\mathbf{s}_{k}=\arg \min _{\mathbf{s} \in K_{l}}\left\|J_{k} \mathbf{s}+\mathbf{f}_{k}\right\|,
$$

where $K_{l}=K_{l}\left(\mathbf{f}_{k}, J_{k}\right)$ is an $l$-dimensional approximate basis for $\operatorname{Col}\left(J_{k}\right)$. Iterations cease when $\left\|J_{k} \mathbf{s}_{k}+\mathbf{f}_{k}\right\|<$ $\delta_{k}$. Moreover, in building $K_{l}$ to solve (18), the inexact Newton-Krylov method only requires directional derivatives, which we approximate using the nonlinear residual function $\mathbf{f}$,

$$
J(\mathbf{U}) \mathbf{V} \approx[\mathbf{f}(\mathbf{U}+\sigma \mathbf{V})-\mathbf{f}(\mathbf{U})] / \sigma, \quad \text { with } \sigma \text { 'small' }
$$

Due to this nesting of iterative algorithms, the solution of the nonlinear problem (17) with sundials only requires encapsulation of a data structure for the solution vector $\mathbf{U}$, user-defined vector operations on $\mathbf{U}$ (e.g. axpy, two-norm, max, etc) and a user-supplied routine for $\mathbf{f}(\mathbf{U})$.

Although it is possible to construct a fully implicit solver out of these relatively simple components, algorithmic scalability with increasing mesh size inherently depends on how rapidly each of these nested iterations converges. It has recently been shown that for a range of PDE problems, the Newton method convergence is mesh independent [8]. However, the basic Krylov iteration requires increasing iterations with mesh refinement $[9,10]$. It is in this context that preconditioners are employed, which strive to accelerate convergence of the Krylov iteration to achieve increased efficiency, robustness and scalability of the overall simulation code.

Prior to describing our preconditioning approaches for this problem, we must first construct the Jacobian entries themselves (since they were not required in (20)). It is in the construction of these preconditioning matrices that we employ tools from automatic differentiation (AD).

\section{Jacobian construction}

Due to the nonlinear complexity of our PDE system (10), the changing spatial stencil between domain interior and boundary zones and a desire to explore preconditioners based on a variety of reduced stencil approximations, analytical derivation of the Jacobian entries proved intractable. Instead, we focused on interfacing our code with the AD tool OPENAD [11-13]. AD tools are typically source code translation programs, wherein a user marks their function arguments as dependent variables, independent variables or parameters, and the AD tool processes this file to produce a new function file that implements the derivative of the dependent variables with respect to the independent variables. While for many applications a hand-coded Jacobian routine can outperform auto-generated routines, AD-produced code is generally error-free, and in many cases does not result in a significant performance penalty over hand-coded routines. Since AD tools are source code translators, traditionally each tool has been specific to a given programming language, with the majority of such tools built for simply structured languages such as ForTRAN77 and C. OPENAD, on the other hand, is designed around a more flexible framework in which the AD engine is language independent, with interfaces that work with Fortran77, Fortran90, $\mathrm{C}$ and $\mathrm{C}++$. Moreover, the Fortran90 interface allows module-based object oriented programming, which our visco-resistive MHD code uses heavily. Finally, OPENAD is open-source, and is actively supported with funding from NASA, DOE and NSF.

In order to prepare our code for $\mathrm{AD}$, we had to first reconfigure our routine for $\mathcal{R}(\mathbf{U})$ to more optimally interface with OPENAD. To construct our Jacobian entries, we perform forward differentiation in vector mode, 

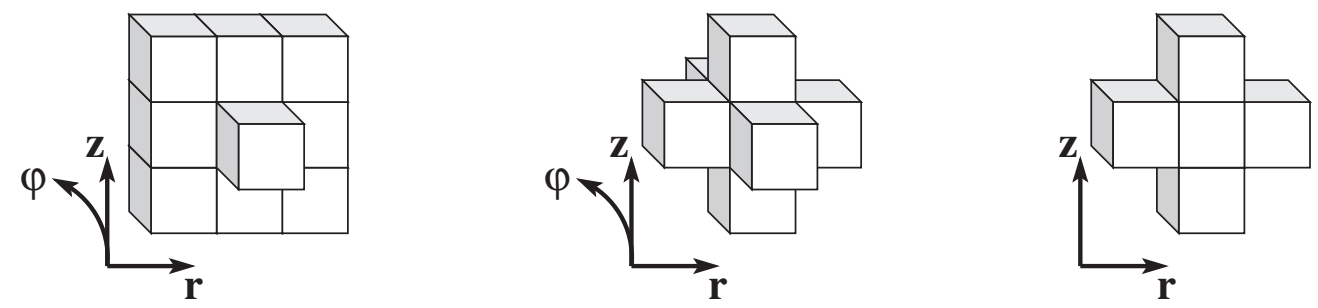

Figure 4. Reduced stencils used in constructing approximate preconditioners: 11-point 3D stencil (left), 7-point 3D stencil (middle) and 5-point 2D stencil (right).

Table 1. Average wall-clock times and numerical accuracy for Jacobian construction approaches (AD $=$ OPENAD; FD = finite difference). All times are reported in seconds, and correspond to the average wall-clock time required per spatial cell in the FV grid. FD error values are averaged over the test grids for each stencil, and are calculated using $\gamma=1$.

\begin{tabular}{lcccc}
\hline Dimension & Stencil & AD time & FD time & FD error \\
\hline 3 & $19 \mathrm{pt}$ & $4.728 \mathrm{e}-4$ & $2.868 \mathrm{e}-4$ & $9.996 \mathrm{e}-5$ \\
3 & $11 \mathrm{pt}$ & $4.201 \mathrm{e}-4$ & $1.452 \mathrm{e}-4$ & $1.579 \mathrm{e}-4$ \\
3 & $7 \mathrm{pt}$ & $3.947 \mathrm{e}-4$ & $8.085 \mathrm{e}-5$ & $1.259 \mathrm{e}-4$ \\
2 & $9 \mathrm{pt}$ & $2.476 \mathrm{e}-4$ & $1.528 \mathrm{e}-4$ & $5.015 \mathrm{e}-6$ \\
2 & $5 \mathrm{pt}$ & $2.165 \mathrm{e}-4$ & $4.887 \mathrm{e}-5$ & $1.652 \mathrm{e}-5$ \\
\hline
\end{tabular}

based on a so-called 'strip-mining' approach, wherein we repeatedly call the auto-generated routine to fill in non-zero entries of our Jacobian matrix. However, since vector-mode AD computes derivative information of an output with respect to all possible inputs, and since our $\mathcal{R}(\mathbf{U})$ is based on spatially local finite-volume stencils, basic usage of OPENAD would result in the calculation of all entries in a given Jacobian matrix row, even though the vast majority of these entries are zero. To circumvent this, we cloned our existing flux routines to instead calculate one spatial location, $\mathbf{x}_{i}$, of $\mathcal{R}$ at a time, based on only the 19-point nearest-neighbor stencil of unknowns, $\tilde{\mathbf{U}}_{i}$. In this process, we had to take special care to properly modify the entries passed into and out of this patch, based on whether $\mathbf{x}_{i}$ is in the domain interior or boundary. Accounting for our eight unknowns per spatial location, this resulted in an input patch $\tilde{\mathbf{U}}_{i}$ with 152 entries, as opposed to an input $\mathbf{U}$ with $8 N_{\xi} N_{\eta} N_{\varphi}$ entries. Armed with this patch-based residual routine, $\tilde{\mathcal{R}}_{i}\left(\tilde{\mathbf{U}}_{i}\right)$, we then used OpENAD to generate a Jacobian computation routine, $\frac{\partial \tilde{\mathcal{R}}_{i}}{\partial \tilde{\mathbf{U}}_{i}}\left(\tilde{\mathbf{U}}_{i}\right)$, which we incorporated into the main code base. In addition to this straightforward Jacobian computation routine, we also generated alternative versions for 2D simulations, as well as versions that used reduced stencil approximations of $\tilde{\mathcal{R}}_{i}\left(\tilde{\mathbf{U}}_{i}\right)$ as shown in figure 4 , so that we could investigate preconditioners formed using a hierarchy of Jacobian approximations of varying cost, complexity and accuracy.

We then compared the resulting OPENAD-generated Jacobians against those formed using simple finitedifference approximations,

$$
[J(\mathbf{U})]_{i, j}=\delta_{i, j}-\frac{\gamma}{\sigma}\left[\tilde{\mathcal{R}}_{i}\left(\tilde{\mathbf{U}}_{i}+\sigma \mathbf{e}_{j}\right)-\tilde{\mathcal{R}}_{i}\left(\tilde{\mathbf{U}}_{i}\right)\right]+\mathcal{O}(\gamma \sigma),
$$

where $\sigma$ was optimally chosen as $10^{-8}$ since the values of $\tilde{\mathbf{U}}_{i}$ are unit-normalized [14]. We then tested these approaches for both speed and accuracy to determine an optimal approach for preconditioner construction in our code. As seen in table 1, while the finite-difference approach was marginally faster than the AD approach, it resulted in a significant loss of accuracy compared with the auto-differentiated routines, which are all accurate to near machine precision.

For a more thorough account of our use of OPENAD in this application, see [15]. 


\section{Preconditioner formulations}

As discussed in section 3.2, a critical component of an efficient and robust Newton-Krylov solver is an effective preconditioner, $P \approx J^{-1}$, to accelerate convergence of the Krylov iteration [9]. We employ the right preconditioner variant,

$$
J \mathbf{s}=-\mathbf{f}, \quad \Leftrightarrow \quad J P P^{-1} \mathbf{s}=-\mathbf{f}, \quad \Leftrightarrow \quad \begin{aligned}
& (J P) \mathbf{w}=-\mathbf{f}, \\
& \mathbf{s}=P \mathbf{w},
\end{aligned}
$$

since it does not change the units of the computed linear residual as is the case with left preconditioning,

$$
J \mathbf{s}=-\mathbf{f}, \quad \Leftrightarrow \quad P J \mathbf{s}=-P \mathbf{f}, \quad \Leftrightarrow \quad \begin{aligned}
& \mathbf{w}=-P \mathbf{f}, \\
& (P J) \mathbf{s}=\mathbf{w} .
\end{aligned}
$$

The focus of this paper is on examining a variety of geometry-based preconditioning approaches that have been inspired by commonly used solvers in current MHD simulation software. Specifically, a variety of MHD codes decouple the poloidal $(\xi, \eta)$-plane and toroidal $(\varphi)$ directions within their implicit formulation, due to the decreased numerical stiffness in the toroidal direction [16-18]. Hence, in this paper we focus on a range of preconditioners that further explore the need for implicit coupling between these directions. However, since our 19-point stencils from figure 3 do not easily decouple into poloidal and toroidal components, we allow approximations to the Jacobian within our preconditioner using the reduced stencils shown in figure 4. For each of our reduced stencil approximations, we transfer non-zero Jacobian entries from corner dependences to face-neighbor dependences, e.g.

$$
\begin{aligned}
& J_{(i, j, k),(i+1, j, k)}=J_{(i, j, k),(i+1, j, k)}+\frac{1}{2} J_{(i, j, k),(i+1, j+1, k)}, \\
& J_{(i, j, k),(i, j+1, k)}=J_{(i, j, k),(i, j+1, k)}+\frac{1}{2} J_{(i, j, k),(i+1, j+1, k)}, \\
& J_{(i, j, k),(i+1, j+1, k)}=0 .
\end{aligned}
$$

\subsection{Alternating direction implicit split preconditioner, $P_{\mathrm{ADI}}$}

The first of our preconditioners uses an ADI-based approach that solves periodic, block-tridiagonal matrices along each of the $\xi, \eta$ and $\varphi$ directions in sequential sweeps. Such approaches rely on an operator splitting of the form

$$
\begin{aligned}
J \mathbf{s} & =\left(I-\gamma\left(J_{1}+J_{2}+J_{3}\right)\right) \mathbf{s} \\
& \approx\left(I-\gamma J_{1}\right)\left(I-\gamma J_{2}\right)\left(I-\gamma J_{3}\right) \mathbf{s} \\
& \equiv P_{\mathrm{ADI}}^{-1} \mathbf{s} .
\end{aligned}
$$

We may then apply the preconditioner, $y=P_{\mathrm{ADI}} z$, using a sequence of three 1D solves,

$$
w=\left(I-\gamma J_{1}\right)^{-1} z \quad \rightarrow \quad x=\left(I-\gamma J_{2}\right)^{-1} w \quad \rightarrow \quad y=\left(I-\gamma J_{3}\right)^{-1} x .
$$

Preconditioners based on this approach are especially attractive in structured grid calculations, since each unidirectional solve can be very efficient/scalable, as shown in figure 5. However, due to their decoupling of $J$ into separate directional components, their accuracy suffers due to missing coupling between directions, since the error term has the form

$$
J-P_{\text {ADI }}^{-1}=-\gamma^{2}\left(J_{1} J_{2}+J_{1} J_{3}+J_{2} J_{3}\right)+\gamma^{3} J_{1} J_{2} J_{3}
$$

Hence, for strongly coupled directions the associated product terms may rapidly outweigh the time stepdependent factor, $\gamma^{2}$, and destroy the benefits of the preconditioning approach.

In the context of our mapped grid equation (10), these directional operators act along the radial $(\xi)$, azimuthal $(\eta)$ and toroidal $(\varphi)$ directions independently, each resulting in a block-tridiagonal system comprised of $8 \times 8$ matrix blocks, corresponding to the dependences of all eight variables in a given finite-volume cell on the variables in the neighboring cell in the associated direction. In the $\eta$ and $\varphi$ directions these systems 


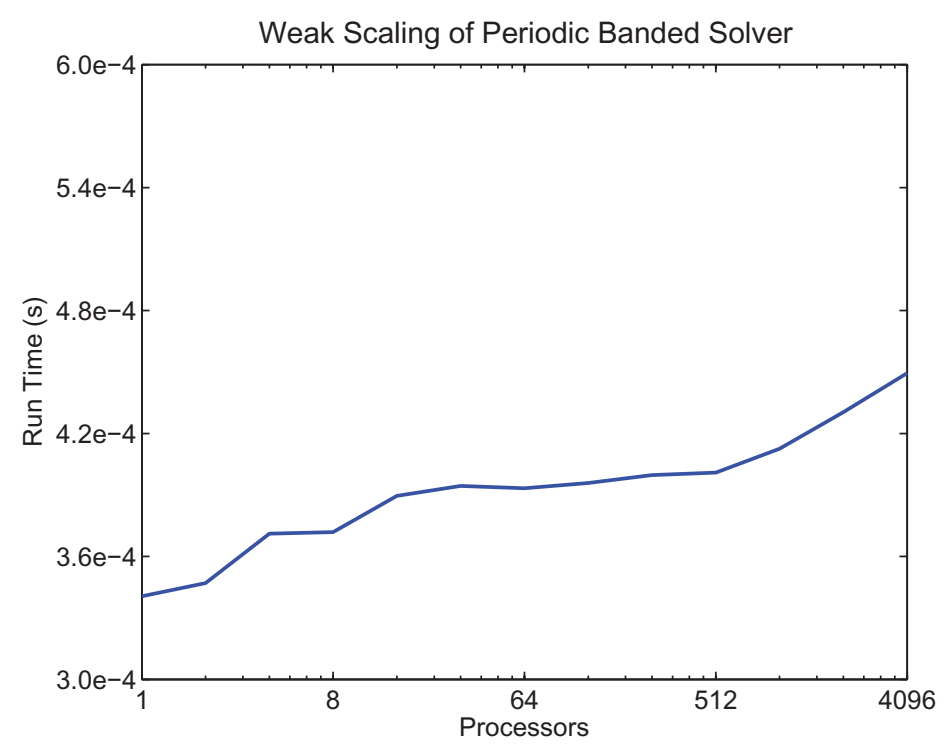

Figure 5. Weak scaling for one uni-directional periodic parallel tridiagonal solve within an ADI-based preconditioner. Each process owns a $64^{3}$ spatial grid, and we increase the number of nodes in one direction. Times shown are averaged over repeated calls. Note the performance degradation by only $35 \%$ from 1 to 4096 processes, with much of this slowdown occurring from 1 to 10 processes.

are periodic. Parallelism is achieved through a pipelining strategy, in which, for example, each of the distinct $N_{\xi} \times N_{\eta}$ systems in the $\varphi$ direction is solved asynchronously using sequential parallel algorithms.

Unfortunately, however, the 19- and 9-point spatial stencils illustrated in figure 3 do not easily decouple into distinct systems in each of the $\{\xi, \varphi, \eta\}$ directions, due to the corner couplings in non-coordinate-aligned directions. This was the impetus for implementing the 7- and 5-point reduced stencil approximations shown in figure 4, which are the approximations used in constructing $P_{\mathrm{ADI}}$.

\subsection{Restricted additive Schwarz preconditioners}

Our second class of preconditioners use a restricted additive Schwarz (RAS) formulation [19]. In this approach, we decompose the linear operator according to the underlying message passing interface (MPI) domain decomposition, and solve the Jacobian components separately on each process. For example, if the overall domain $\Omega$ can be written as the union of $p$ non-overlapping subdomains $\Omega=\bigcup_{i=1}^{p} \Omega_{i}$, then a simple block Jacobi preconditioner would approximate

$$
J^{-1} \approx \sum_{i=1}^{p} R_{i}^{\mathrm{T}} J_{i}^{-1} R_{i},
$$

where each $R_{i}$ is a restriction operator taking values from the full domain $\Omega$ to the subdomain $\Omega_{i}$, and $J_{i}$ is the portion of the Jacobian corresponding to couplings between $\Omega_{i}$ and itself (i.e. decoupled from all other $\Omega_{j}, j \neq i$ ). The accuracy of this approach suffers due to its inability to propagate information from one subdomain to the next. Additive Schwarz (AS) methods consider expanding each subdomain to include a small overlap with neighboring subdomains. These expanded subdomains $\tilde{\Omega}_{i}$ typically include ghost zones already held on each processor for MPI communication. In AS methods, one then approximates

$$
J^{-1} \approx \sum_{i=1}^{p} \tilde{R}_{i}^{\mathrm{T}} \tilde{J}_{i}^{-1} \tilde{R}_{i},
$$

where these new components are defined on the enlarged subdomains $\tilde{\Omega}_{i}$, and the solution in the overlap regions receives contributions from multiple processes in an additive fashion. A more communication-efficient 

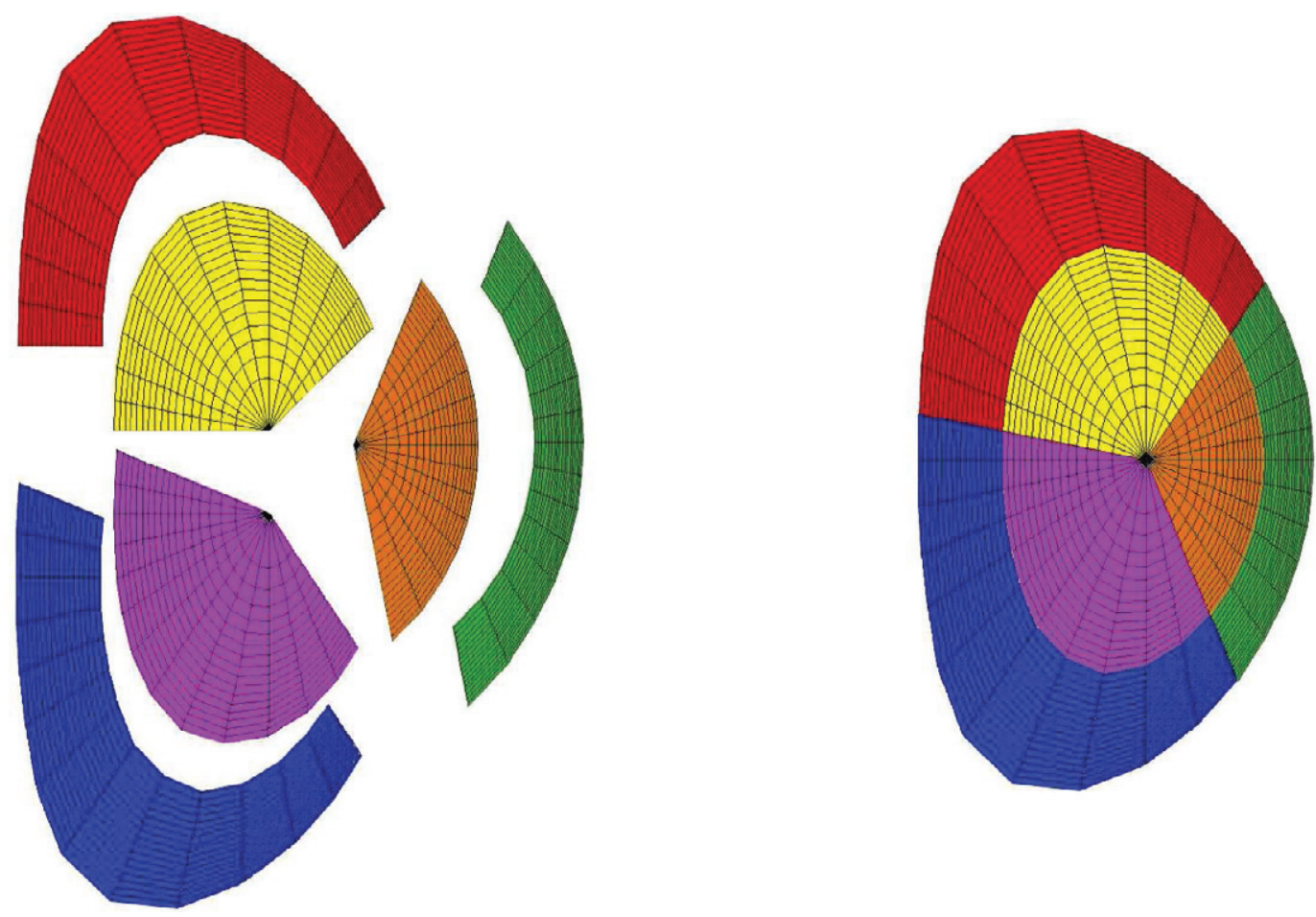

Figure 6. Schematic diagram of an overlapping RAS solve on the poloidal plane among six processes. Although each process solves a local problem on its enlarged subdomain (left), the eventual solutions are only retained for the portion of the domain owned by each process (right). Here, the overlap width between subdomains consists of two cells at each interface.

variant of AS, which has also been shown to result in a provably higher rate of convergence, is the RAS algorithm $[19,20]$, which replaces the prolongation matrix $\tilde{R}_{i}^{\mathrm{T}}$ with a variant $\hat{R}_{i}^{\mathrm{T}}$ wherein each enlarged subdomain $\tilde{\Omega}_{i}$ only contributes to the solution defined on its restricted subdomain $\Omega_{i}$, i.e.

$$
P_{\mathrm{RAS}}=\sum_{i=1}^{p} \hat{R}_{i}^{\mathrm{T}} \tilde{J}_{i}^{-1} \tilde{R}_{i} .
$$

A schematic diagram of this idea is shown in figure 6.

In solving the local subdomain problems, $\tilde{J}_{i}^{-1}$, we use the SUPERLU solver package that implements a highly efficient sparse direct $L U$ factorization algorithm. These Jacobians are stored in compressed sparse row (CSR) format, using a drop tolerance of $10^{-10}$ for a conservative pruning of small entries from the resulting matrix. This CSR matrix is then passed through a custom-built ForTRAN90/ C interface to call SUPERLU.

The RAS preconditioning approach can also benefit from the reduced stencil approximations shown in figure 4, since a decreased stencil results in lower CSR memory requirements, decreased fill-in within the $L U$ factorization and increased factorization speed. Of course, the detractor in any reduced stencil approximation is that the preconditioner only relates to the true Jacobian in an approximate sense, thereby potentially slowing the Krylov convergence. We therefore consider the following set of RAS-based preconditioners:

- $P_{\text {RAS }}$ uses the full 19-point stencil on each subdomain.

- $P_{\mathrm{RAS} p}$ uses only a 9-point stencil in each poloidal $(\xi, \eta)$ plane, i.e. it does not precondition in the $\varphi$ direction at all, similarly to the implicit solvers in [16-18].

- $P_{\mathrm{RAS} p 5}$ uses only a reduced 5-point stencil in each poloidal $(\xi, \eta)$ plane, ignoring $\varphi$ coupling and $\xi-\eta$ corner coupling.

In addition, we consider varying overlap widths, with a default of two cells at each interface unless specified otherwise. 


\subsection{Split poloidal/toroidal preconditioners}

Our third preconditioning approach is a hybrid of the two previous ones, and attempts to investigate the question of whether an implicit solve that ignores the $\varphi$ direction is problematic at larger scales or with finer resolutions. This preconditioner therefore attempts to fill the gap between a fully coupled 3D preconditioning strategy (as in $P_{\mathrm{RAS}}$ ) and a solve within only the poloidal plane (as in $P_{\mathrm{RAS} p}$ and $P_{\mathrm{RAS} p 5}$ ). In this hybrid approach, we employ our overlapping RAS solver for poloidal subsystems, and follow up with a parallel, periodic, block-tridiagonal solve in the $\varphi$ direction only. We define two such preconditioners,

$$
\begin{aligned}
& P_{H 11}=\left(I-\gamma J_{\varphi}\right)^{-1} P_{\mathrm{RAS} p}, \\
& P_{H 7}=\left(I-\gamma J_{\varphi}\right)^{-1} P_{\mathrm{RAS} p 5} .
\end{aligned}
$$

The numbering scheme (11 versus 7) corresponds to which approximate stencil from figure 4 is used in constructing these preconditioner components.

Our hypothesis is that the resulting hybrid approaches will benefit from increased speed over $P_{\mathrm{RAS}}$, due to the decoupling of each poloidal plane in the $\varphi$ direction. Moreover, it will benefit from increased accuracy over $P_{\mathrm{ADI}}$ due to its treatment of the tight coupling between the $\xi$ and $\eta$ directions resulting from our mapped formulation. However, the key question will be to compare the performance of $P_{H 11}$ and $P_{H 7}$ against their poloidal-only counterparts, $P_{\mathrm{RAS} p}$ and $P_{\mathrm{RAS} p 5}$, which ignore implicit contributions in the $\varphi$ direction completely.

\section{Numerical results}

We examine the above preconditioning strategies on a 3D pellet injection fueling model problem. For this problem, we use reflecting boundary conditions at the outer, $\xi=\xi_{\max }$, wall, no flux conditions at the inner, $\xi=\xi_{\min }$, core and periodic boundary conditions in both the $\eta$ and $\varphi$ directions. We initialize the plasma in an analytical Solovev equilibrium configuration according to Bateman [21],

$$
\psi(R, Z)=\frac{p^{\prime}}{2\left(1+\alpha^{2}\right)}\left(\left(R^{2}-\gamma\right) Z^{2}+\frac{\alpha^{2}}{4}\left(R^{2}-R_{0}^{2}\right)^{2}\right)
$$

where we use the parameters $\gamma=p^{\prime}=\frac{1}{4}, \alpha=\sqrt{2}$ and $R_{0}=1$. To this equilibrium we add a small region of high density and pressure to represent a recently ablated pellet launched from the high field side,

$$
\rho(\mathbf{x})=1+1000 \mathrm{e}^{-600\left|\mathbf{x}-\mathbf{x}_{0}\right|^{2}} \quad \text { and } \quad p(\mathbf{x})=p_{0}(\mathbf{x}) \rho(\mathbf{x}),
$$

where $x_{0}$ is the pellet center, and $p_{0}$ is the pressure prior to perturbation. We employ two sets of diffusive parameters: our primary tests run with a Lundquist number of $10^{3}$, and we also test a harsher regime with this set to $10^{4}$. Both regimes use a thermal Prandtl number of 0.7 and a magnetic Prandtl number of 1.0. Since the initial transport of the pellet mass is one of the most difficult phases of this calculation and we are using this problem primarily for testing preconditioner efficacy, all tests evolve the problem for a short duration of 0.1 Alfvén time unit $\left(t_{\mathrm{a}}=\frac{1}{v_{\mathrm{a}}}\right)$, using a time step of $0.05 t_{\mathrm{a}}$, where the Alfvén velocity is $v_{\mathrm{a}}=\frac{1}{\sqrt{\mu_{0} \rho_{0}}}$, the reference plasma density is $\rho_{0}=3.321 \times 10^{-8}$ and $\mu_{0}=4 \pi \times 10^{-7}$ is the permeability of free space.

We use the $\theta$-method for time discretization, with $\theta=1$. Non-default input parameters for the KINSOL inexact Newton-Krylov solver within sundiaLs are summarized in table 2. The linear systems (19) use up to 200 BiCG-Stab iterations for memory efficiency, with the side effect that for very poorly preconditioned problems these iterations may not converge. Since these tests run for only a few time steps, we only set up and factor the preconditioning matrix once upon initialization, and reuse this initial preconditioner through subsequent nonlinear iterations and time steps, to amortize the cost of preconditioner construction (moderate) and factorization (significant). 
Table 2. Non-default parameters used within the kinsol nonlinear solver.

\begin{tabular}{ll}
\hline Parameter & Value \\
\hline KINSetEtaForm & KIN_ETACONSTANT \\
KINSetEtaConstValue & $1 \mathrm{e}-3$ \\
KINSetNumMaxIters & 5 \\
KINSetMaxSubSetupCalls & 1 \\
KINSetFuncNormTol & $1 \mathrm{e}-5$ \\
KINSetScaledStepTol & $1 \mathrm{e}-9$ \\
strategy & KIN_NONE \\
\hline
\end{tabular}

We first investigated the preconditioning approaches $P_{\mathrm{ADI}}, P_{\mathrm{RAS}}, P_{\mathrm{RAS} p}, P_{\mathrm{RAS} p 5}, P_{H 11}$ and $P_{H 7}$, where the latter five solvers used an overlap width of 2 at each face, against a solver without preconditioning, on serial tests using meshes with $16 \times 16 \times 16,32 \times 32 \times 16$ and $64 \times 64 \times 16$ cells $\left(N_{\xi} \times N_{\eta} \times N_{\varphi}\right)$. These initial exploratory tests were used to give an idea of preconditioner performance on smaller scale problems, to properly guide tests at larger scales. Results showing average Krylov iterations per Newton step, and average wall-clock timings per Newton step per mesh cell, are shown in figure 7.

As can be seen in these tests, the pure 3D RAS solver is only effective on small-scale problems, due to its significant memory requirements and factorization costs as the local matrix on each process grows in size. Moreover, the pure ADI solver is only effective on the high Lundquist problems, most likely due to the fact that the non-coordinate-aligned stencil couplings derive from the visco-resistive terms. Hence, on problems with non-negligible diffusive terms, the pure ADI preconditioner requires a large number of Krylov iterations as the mesh is refined, due to its inherent decoupling between the $\xi$ and $\eta$ directions within each poloidal plane. Because of these results, we do not consider either the pure $P_{\mathrm{ADI}}$ or $P_{\mathrm{RAS}}$ approaches in subsequent tests.

In addition to ruling out these two limiting options, we see that the un-preconditioned solver requires significantly more Krylov iterations than its preconditioned rivals, but due to its reduced complexity remains competitive in run time. Finally, we see that the differences between the poloidal-only RAS preconditioners, $P_{\mathrm{RAS} p}$ and $P_{\mathrm{RAS} p 5}$, and the hybrid preconditioners, $P_{H 11}$ and $P_{H 7}$, are too small to distinguish on such smallscale problems.

We then investigated the preconditioning approaches $P_{\mathrm{RAS} p, 2}, P_{\mathrm{RAS} p, 4}, P_{\mathrm{RAS} p 5,2}, P_{\mathrm{RAS} p 5,4}, P_{H 11,2}$, $P_{H 11,4}, P_{H 7,2}$ and $P_{H 7,4}$ on parallel tests, comparing them against each other and the un-preconditioned solver. We ran the RAS and hybrid solvers using overlap widths of both 2 and 4, adding the suffix ',2' or ',4' to the preconditioner names as appropriate. The goal of these tests was to further explore three questions:

(a) How does the RAS overlap width play a role in preconditioner efficacy?

(a) How much do stencil approximations in a preconditioner affect its utility?

(c) How important is the inclusion of toroidal effects in the preconditioner?

To this end, we tried these preconditioners in both normal and high Lundquist regimes in a weak scaling scenario, using a base grid of $32 \times 32 \times 16$ cells per processor, with processor decompositions $\left(P_{\xi}, P_{\eta}, P_{\varphi}\right) \in$ $\{(1,1,1),(1,2,1),(2,2,1),(2,4,1),(2,4,2),(4,4,2),(4,8,2),(4,8,4)\}$. The average number of Krylov iterations per Newton step for each test are shown in figure 8. In order to distinguish between the preconditioned results, the vertical axis limits are reduced to a maximum of 8. As a result, the nonpreconditioned solver is not visible on the plots, since it required $\{15.6,17.8,35.8,33.8,44.0,95.9,105.6\}$ average Krylov iterations for the standard regime and $\{32.0,40.0,101.9,107.8,120.1\}$ average Krylov iterations for the high-Lundquist-number regime. We note that this non-preconditioned solver could not converge for the two largest test problems in the higher-Lundquist-number regime.

With this accuracy-based measure of a preconditioner's utility, we may address our questions above. To examine the effect of RAS overlap width in these tests, we compare the solid and dashed lines having corresponding symbols. In nearly every case, the dashed line (overlap of 4) requires fewer Krylov iterations 

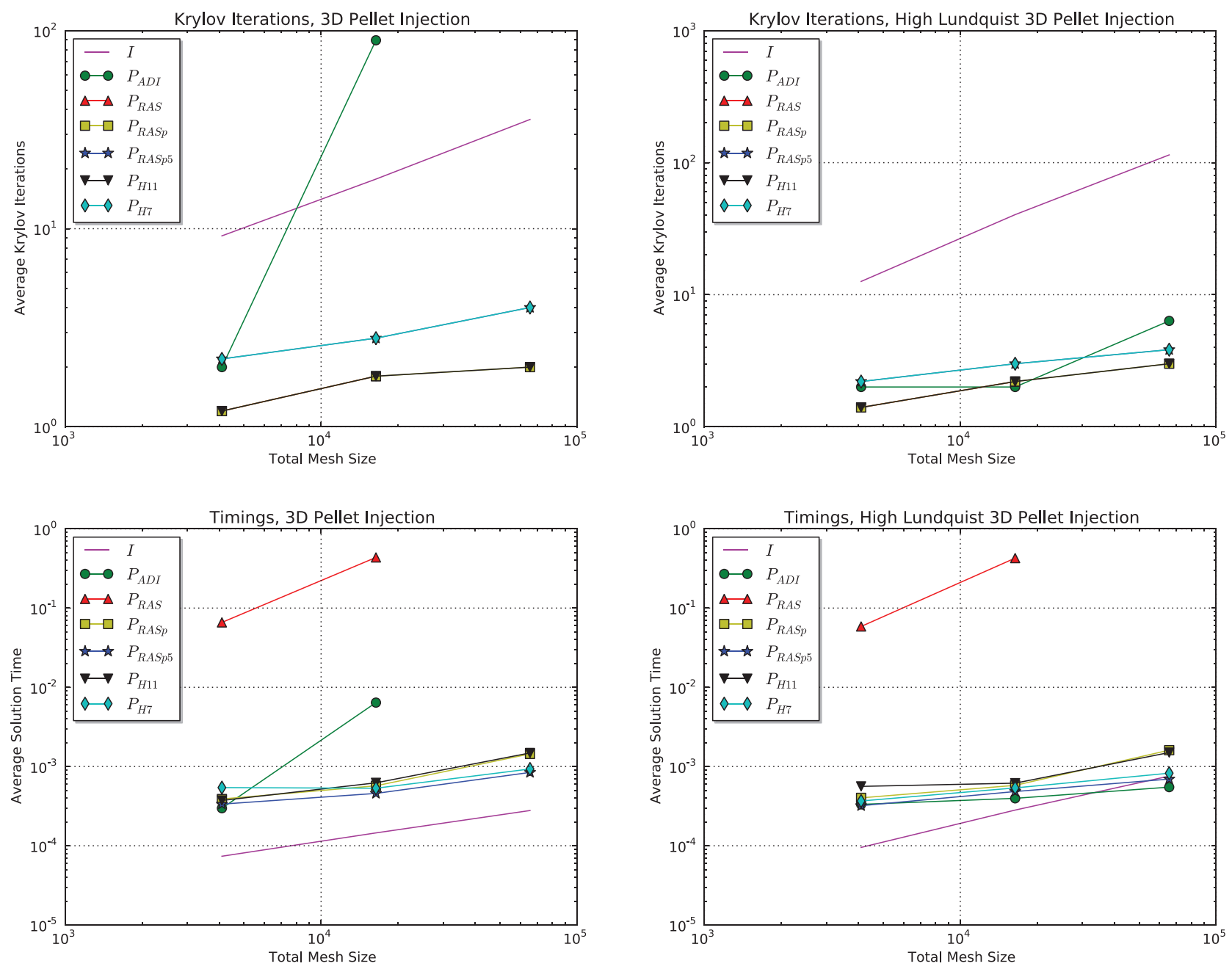

Figure 7. Krylov iterations and averaged timings for serial tests on the preconditioners $I, P_{\mathrm{ADI}}, P_{\mathrm{RAS}}$, $P_{\mathrm{RAS} p}, P_{\mathrm{RAS} p 5}, P_{H 11}$ and $P_{H 7}$. The three mesh sizes were $16 \times 16 \times 16,32 \times 32 \times 16$ and $64 \times 64 \times 16$. The top row shows the average number of Krylov iterations per Newton step; the second row shows the average times per mesh cell per Newton step. The plots on the left use Reynolds and Lundquist numbers of $10^{3}$, and those on the right use $10^{4}$. Note that $P_{\mathrm{ADI}}$ did not converge for the finest mesh on the lower Lundquist number test. $P_{\text {RAS }}$ failed due to running out of memory $(>44 \mathrm{~GB})$ for the finest mesh on both regimes, and had much slower run times due to the more costly factorization stage.

than its solid counterpart (overlap of 2), with this effect more dramatically seen in the higher-Lundquistnumber regime. This result is as expected, since an increasing overlap allows further interaction between neighboring processes within each preconditioner solve.

Our second question, regarding the effect of reduced stencil approximations on preconditioner accuracy, may be understood by comparing $P_{\mathrm{RAS} p}$ (circles) against $P_{\mathrm{RAS} p 5}$ (triangles), and $P_{H 11}$ (squares) against $P_{H 7}$ (stars). In all cases, the use of the reduced stencil approximation resulted in significantly more Krylov iterations than the full stencil. This effect is most pronounced in the standard parameter regime, wherein the full stencil preconditioners required approximately half the Krylov iterations compared with the reduced approximations. This may be easily understood due to the fact that the increased stencil sizes are due to the diffusive terms in the visco-resistive terms, which play a smaller role in the higher-Lundquist-number regime.

Lastly, on the question of the need to include toroidal effects in a preconditioner, we compare the $P_{H 11}$ and $P_{\mathrm{RAS} p}$ lines (squares versus circles) or the $P_{H 7}$ and $P_{\mathrm{RAS} p 5}$ lines (stars versus triangles). At all mesh sizes the hybrid preconditioners perform as well as or better than poloidal-only solvers. However, this difference is 

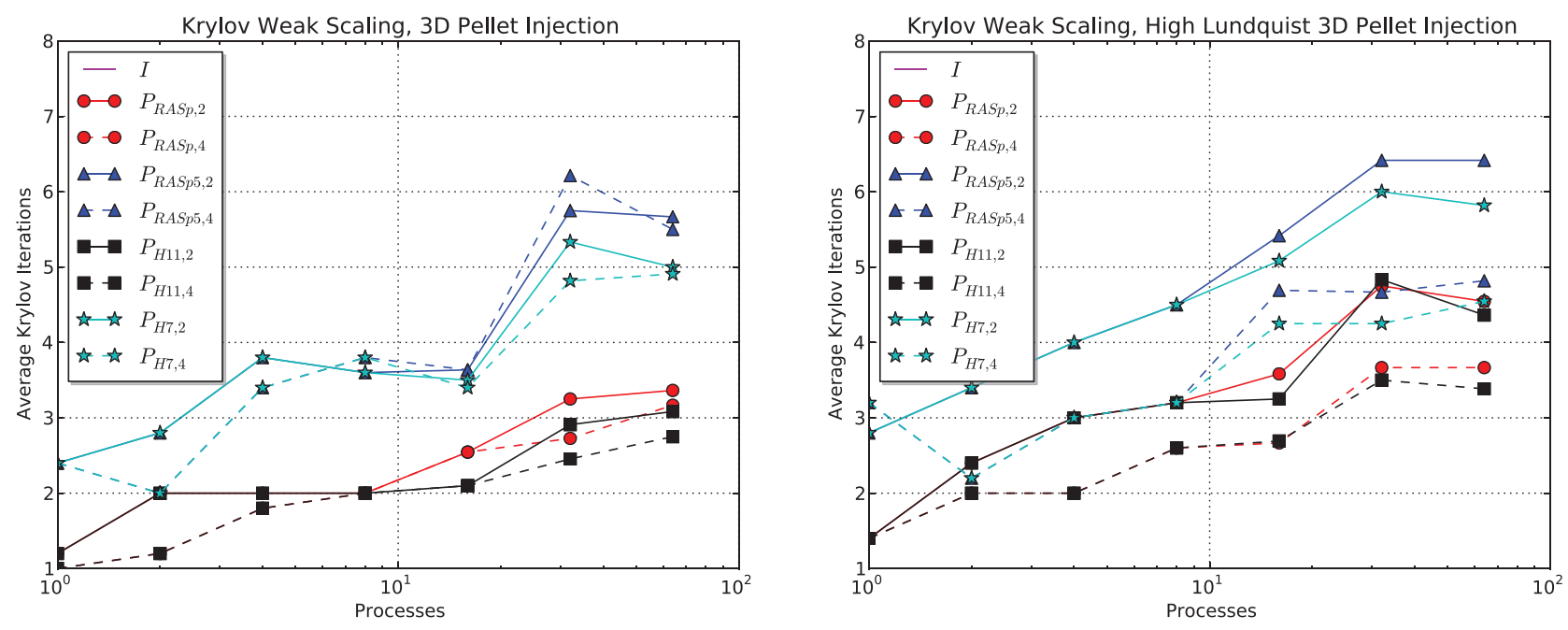

Figure 8. Krylov weak scaling tests: the average number of Krylov iterations per Newton step is plotted as a function of mesh size/parallelism. The left uses Reynolds and Lundquist numbers of $10^{3}$ and the right uses $10^{4}$. Each process owns $32 \times 32 \times 16$ cells. The non-preconditioned solver iterations did not fit on the plots, and had values $\{15.6,17.8,35.8,33.8,44.0,95.9,105.6\}$ for the left plot and $\{32.0,40.0,101.9,107.8,120.1\}$ for the right plot.
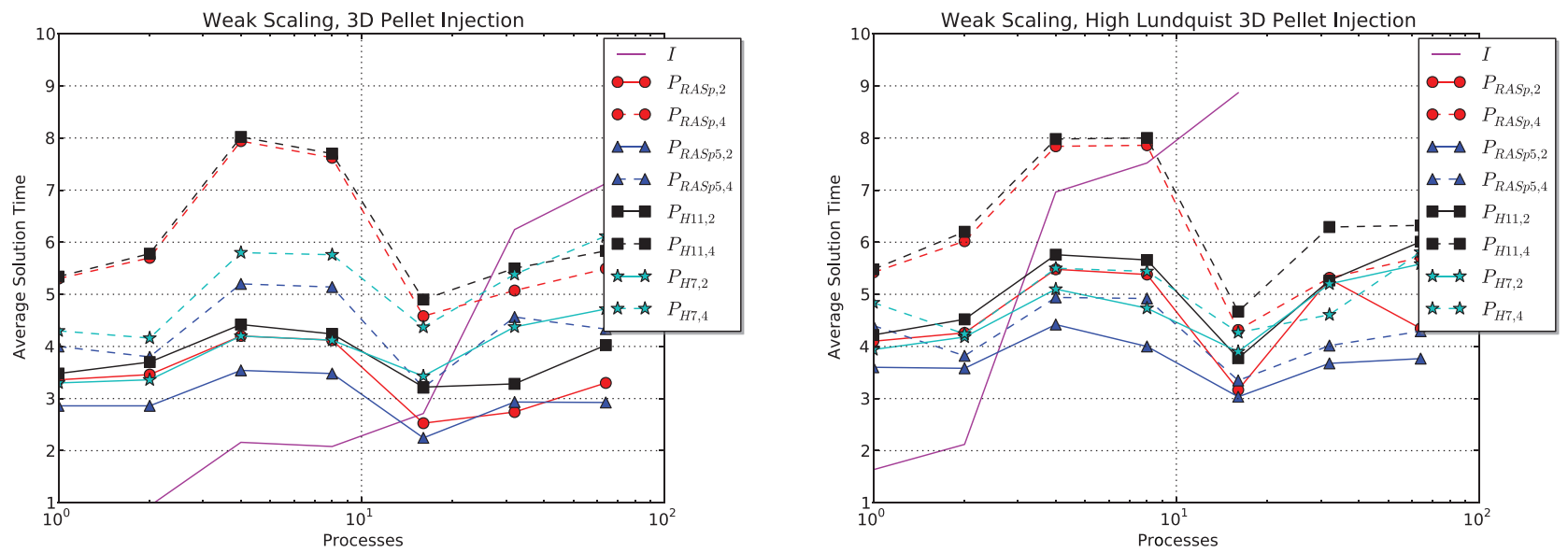

Figure 9. Weak scaling times: the average wall-clock time per Newton step is plotted as a function of mesh size/parallelism. The left uses Reynolds and Lundquist numbers of $10^{3}$ and the right uses $10^{4}$. Each process owns $32 \times 32 \times 16$ cells. Note that the non-preconditioned solver line stops in the plot at the right, indicating solver divergence with increasing mesh size.

small, and seems to be much less important to preconditioner accuracy than either overlap width or reduced stencil approximations.

However, a reduction in Krylov iterations is not entirely indicative of the total efficiency of a solver, since each preconditioner will have different computational costs per solve. Due to the differing complexities of each method, one would anticipate that the cost of a single preconditioner solve would be lowest for $P=I$ and would increase in the order $P_{\mathrm{RAS} p 5,2}, P_{\mathrm{RAS} p 5,4}, P_{\mathrm{RAS} p, 2}, P_{\mathrm{RAS} p, 4}, P_{H 7,2}, P_{H 7,4}, P_{H 11,2}$ and $P_{H 11,4}$. We therefore consider the average solution time per Newton iteration in figure 9 , which gives a more holistic view of the preconditioner efficacy, as it combines both the number of Krylov iterations required for convergence (accuracy) and the speed of each such iteration (efficiency).

With this metric, we see that the average number of Krylov iterations per Newton step only tells a piece of the story. Since the non-preconditioned solver is by far the least expensive per iteration, it is unsurprising that for small-scale problems this solver is faster than all preconditioned options. However, this lack of 
Table 3. Average Krylov iteration counts and wall-clock times per Newton step for 3D simulations using $P_{\mathrm{RAS}, o 4}$ and $P_{H 11, o 4}$, investigating the effect of using the 11-point reduced stencil approximation instead of the full 19-point approach.

\begin{tabular}{cccccc}
\hline Mesh & Processors & $P_{H 11, o 4}$ Krylov & Time & $P_{\text {RAS }, o 4}$ Krylov & Time \\
\hline $32 \times 16 \times 16$ & $1 \times 1 \times 1$ & 1.0 & 5.34 & 1.2 & 1326 \\
$32 \times 32 \times 16$ & $1 \times 2 \times 1$ & 1.2 & 5.78 & 2.0 & 1456 \\
$64 \times 32 \times 16$ & $2 \times 2 \times 1$ & 1.8 & 8.02 & 2.0 & 1748 \\
$64 \times 64 \times 32$ & $2 \times 4 \times 2$ & 2.1 & 4.90 & 2.1 & 961 \\
\hline
\end{tabular}

preconditioning becomes problematic as the mesh size grows, with the resulting solver rapidly surpassing the times required by preconditioned solvers, and even failing altogether for moderate problem sizes in the higher Lundquist regime.

A second general item to note is that aside from a strange increase in run times when using 4 and 8 processes, all of the preconditioned solvers take a relatively constant amount of time per Newton iteration as the problem size grows. This is largely due to the fact that even in our lower Lundquist number tests, the visco-resistive MHD system is overwhelmingly dominated by the stiff hyperbolic terms, on which RASbased preconditioners have traditionally proven effective [19,22]. What remains to be seen is how well these preconditioners perform as the problem sizes increase to larger scales. We plan to investigate these effects with future studies, and hypothesize that above some threshold, these RAS-based solvers will require a coarse grid correction to handle longer-distance interactions than simple nearest-neighbor coupling through overlap regions.

Regarding our specific questions, we see that in all cases, the reduction in Krylov iterations due to an increased overlap width is nullified by its increased computational cost, with the solid lines (overlap 2) uniformly outperforming their dashed line (overlap 4) counterparts. We also see that the increased efficiency of the reduced stencil approximations over their full stencil counterparts quickly compensates for their higher Krylov iteration count, with all triangles outperforming circles, and all stars outperforming squares. Finally, we see that there is little difference in efficiency between the poloidal-only solves and their hybrid poloidal + toroidal counterparts (circle versus square, and triangle versus star). This is largely due to the fact that these solvers required nearly identical numbers of Krylov iterations, and the additional toroidal solve for $P_{H 11}$ and $P_{H 7}$ is significantly more efficient than any of the poloidal solves.

Finally, to better understand the effect of using the 11-point stencil approximation within our most accurate hybrid preconditioner, $P_{H 11,4}$, as compared with a full 19 -point $3 \mathrm{D}$ RAS preconditioner, $P_{\mathrm{RAS}, 4}$, we ran a few more tests. Unfortunately, due to its increased 19-point stencil size and its extents outside the poloidal plane, $P_{\mathrm{RAS}, 4}$ required a large amount of memory, and we could only perform a subset of representative tests with that approach, the results of which are shown in table 3. From these results, it is clear that due to the reduced stiffness of the visco-resistive MHD system in the toroidal direction, neither the 11-point approximation or the split solve between the poloidal plane and the toroidal direction reduced the efficacy of the preconditioner. In addition, due to the significantly more desirable structure of the $P_{H 11}$ matrix as compared with $P_{\text {RAS }}$, the resulting code benefits greatly from both increased speed and decreased memory requirements.

\section{Conclusions and future work}

From the computational results shown in section 6, it is our conclusion that while preconditioning is indeed necessary for a robust, fully implicit solution of this mapped grid visco-resistive MHD problem, the most effective preconditioning approaches employ simplifying approximations designed to decrease their resulting memory and factorization requirements. As a result, the most efficient preconditioning approach overall was $P_{\mathrm{RAS} p 5,2}$, which first approximates the full 19-point 3D stencil using a simple 5-point $2 \mathrm{D}$ approximation within each poloidal plane and then solves the resulting systems using a RAS solve with an overlap width of 2 . Although these approximations resulted in that approach taking the most Krylov iterations per Newton step 
of all the competing approaches, their increased efficiency proved more important, and resulted in the most efficient overall solver. However, the inclusion of an additional solve to incorporate toroidal effects did not significantly slow things down in $P_{H 7,2}$, and could allow increased flexibility when solving problems with more significant toroidal stiffness.

Furthermore, these results hint at optimizations that may be used to achieve additional improvements over those used here. In this formulation, we perform a direct solver using a full $L U$ decomposition within the poloidal plane on each processor. Since the preconditioner only needs to approximate the underlying problem, this $L U$ factorization could be replaced with an incomplete $L U$ factorization, which both minimizes memory requirements and increases the efficiency. Moreover, since the dominant cost of each Schwarz-based preconditioner is in the factorization stage, the tests in this paper performed this factorization only once, and reused it over the course of the (short) simulation. However, in longer production runs as the problem dynamics evolve, this preconditioner can grow stale, no longer representing a solver for the current linearized Newton systems. Instead, one could devise an adaptive strategy for when to recompute and factor the preconditioner, attempting to balance the large factorization time against increased solution times due to reusing a stale $P$.

In addition to these mathematically inspired optimizations, a variety of computer science-related optimizations may prove worthwhile. In this code, we have focused our usage of OpEnAD to be flexible, allowing the full range of 19-, 11-, 9-, 7- and 5-point stencils. With a final decision on the form of approximate stencil to use in the preconditioner, we can tune our use of OPENAD to generate derivative information for the exact stencil size used, instead of the maximum possible size. In addition, the code produced by OPENAD is written for serial or distributed-memory parallel execution, but is comprised of operations that may benefit greatly from threaded parallelism, allowing the use of hybrid MPI and OpenMP programming approaches, as will be increasingly required on upcoming computing hardware.

Finally, the tests performed here reflect relatively modest parameter values for tokamak plasmas; state-ofthe-art supercomputer applications currently push the Lundquist number to $\mathcal{O}\left(10^{6}\right)$ or higher, corresponding to plasmas that are even more strongly dominated by hyperbolic stiffness as compared to viscous and resistive effects. We anticipate that our RAS and hybrid preconditioning approaches will perform well in these largerscale regimes, since the reduced diffusion will result in increasingly localized spatial effects that are accurately captured using RAS solvers. Such simulations will form the focus of our future work in pushing our methods to larger scales.

\section{Acknowledgments}

DRR and HCT were supported, for this study, by the US Department of Energy, under grants DOEER25785 and LBL-6925354. RS was supported by the King Abdullah University of Science and Technology. Simulations in this paper were conducted using the resources of the Center for Scientific Computation, Southern Methodist University, Dallas, TX, USA.

\section{References}

[1] Reynolds D R, Samtaney R and Woodward C S 2006 A fully implicit numerical method for single-fluid resistive magnetohydrodynamics J. Comput. Phys. 219 144-62

[2] Reynolds D R, Samtaney R and Woodward C S 2010 Operator-based preconditioning of stiff hyperbolic systems SIAM J. Sci. Comput. 32 150-70

[3] Jardin S C 2012 Review of implicit methods for the magnetohydrodynamic description of magnetically confined plasmas J. Comput. Phys. 231 822-38

[4] Chacón L 2008 An optimal, parallel, fully implicit Newton-Krylov solver for three-dimensional viscoresistive magnetohydrodynamics Phys. Plasmas 1556103

[5] Breslau J A and Jardin S C 2003 A parallel algorithm for global magnetic reconnection studies Comput. Phys. Commun. 151 8-24

[6] Lütjens H and Luciani J-F 2010 XTOR-2F: a fully implicit Newton-Krylov solver applied to nonlinear 3D extended MHD in tokamaks J. Comput. Phys. 229 8130-43 
[7] Hindmarsh A C, Brown P N, Grant K E, Lee S L, Serban R, Shumaker D E and Woodward C S 2005 SUNDIALS, suite of nonlinear and differential/algebraic equation solvers ACM Trans. Math. Softw. 31 363-96

[8] Weiser M, Schiela A and Deuflhard P 2005 Asymptotic mesh independence of Newton's method revisited SIAM J. Numer. Anal. 42 1830-45

[9] Knoll D A and Keyes D E 2004 Jacobian-free Newton-Krylov methods: a survey of approaches and applications $J$. Comput. Phys. 193 357-97

[10] Keyes D E, Reynolds D R and Woodward C S 2006 Implicit solvers for large-scale nonlinear problems J. Phys.: Conf. Ser. 46 433-42

[11] Utke J 2011 OpENAD, http://www.mcs.anl.gov/OpenAD

[12] Utke J, Naumann U, Fagan M, Tallent N, Strout M, Heimbach P, Hill C and Wunsch C 2008 OpENAD/F: a modular, open-source tool for automatic differentiation of ForTran codes ACM Trans. Math. Softw. 34 18:1-36

[13] Utke J, Naumann U and Lyons A 2011 OpenAD/F: User Manual Technical Report Argonne National Laboratory, http://www.mcs.anl.gov/OpenAD/openad.pdf

[14] Kelley C T 1995 Iterative Methods for Linear and Nonlinear Equations (Frontiers Appl. Math. (Philadelphia:SIAM))

[15] Reynolds D R and Samtaney R 2012 Sparse Jacobian construction for mapped grid visco-resistive magnetohydrodynamics Recent Adv. Algorithmic Differentiation (Lecture Notes Comput. Sci. vol 87) eds S Forth et al (Berlin: Springer) (in press) http://www.springer.com/mathematics/computational+science+\%26+engineering/book/9783-642-300-22-6

[16] Park W, Belova E V, Fu G Y, Tang X Z, Strauss H R and Sugiyama L E 1999 Plasma simulation studies using multilevel physics models Phys. Plasmas 6 1796-803

[17] Jardin S C, Ferraro N, Luo X, Chen J, Breslau J, Jansen K E and Shephard M S 2008 The M3D-C1 approach to simulating 3D 2-fluid magnetohydrodynamics in magnetic fusion experiments J. Phys.: Conf. Ser. 125

[18] Sovinec C R, Gianakon T A, Held E D, Kruger S E, Schnack D D and the NIMROD Team 2003 NIMROD: a computational laboratory for studying nonlinear fusion magnetohydrodynamics Phys. Plasmas 101727

[19] Cai X-C and Sarkis M 1999 A restricted additive Schwarz preconditioner for general sparse linear systems SIAM J. Sci. Comput. 21 792-7

[20] Efstathiou E and Gander M J 2003 Why restricted additive Schwarz converges faster than additive Schwarz BIT Numer. Math. 43 945-59

[21] Bateman G 1978 MHD Instabilities (Cambridge, MA: MIT Press)

[22] Cai X-C, Farhat C and Sarkis M 1998 A minimum overlap restricted additive Schwarz preconditioner and applications in 3d flow simulations Contemp. Math. 218 479-85 